\section{What rheumatologist should know about Fabry disease}

Fabry disease (FD) is a rare X-linked lysosomal storage disease resulting from the deficient activity of the $\alpha$-galactosidase A enzyme and leading to a progressive accumulation of glycosphingolipids in a wide range of cells throughout the body. Early symptoms of FD, that is, neuropathic pain (pain in hands and/or feet characterised by exacerbations that are provoked by fever, exercise or heat), angiokeratoma (clustered angiomas in characteristic areas including bathing trunk area, lips and umbilicus) (figure 1), hypohidrosis/anhidrosis and gastrointestinal disorders, are usually present from childhood or adolescence, while progressive nephropathy, left ventricular hypertrophy (figure 2) and stroke/transient ischaemic attacks develop by the second to fourth decades of life (earlier in males than in females). ${ }^{1}$ Undiagnosed patients with FD can be detected by screening in at-risk populations, such as patients with end-stage renal disease, unexplained myocardial hypertrophy or early stroke (at the age of 18 to 55 years). ${ }^{2}$

Acroparesthesias in FD can be confused with joint pain. Therefore, patients with unexplained arthritis can represent another group of at-risk that may be suitable for screening for FD. In a recent study, Vordenbäumen et al performed GLA sequencing in 798 adult patients with early undifferentiated arthritis (clinical arthritis for $\leq 26$ weeks in $\geq 2$ joints, or 1 joint with morning stiffness $>30 \mathrm{~min}) .{ }^{3}$ Only eight variants of GLA gene were found, none of which was considered to be pathogenic for FD. The authors did not indicate the age range of patients, which were included in the screening study. In our opinion, screening for FD may be justified in adolescents and younger adults with unexplained joint pain, while it would be useless in the middleaged or elderly patients. Nevertheless, we agree with the authors that rheumatologists should be alert to the differential diagnosis of FD.

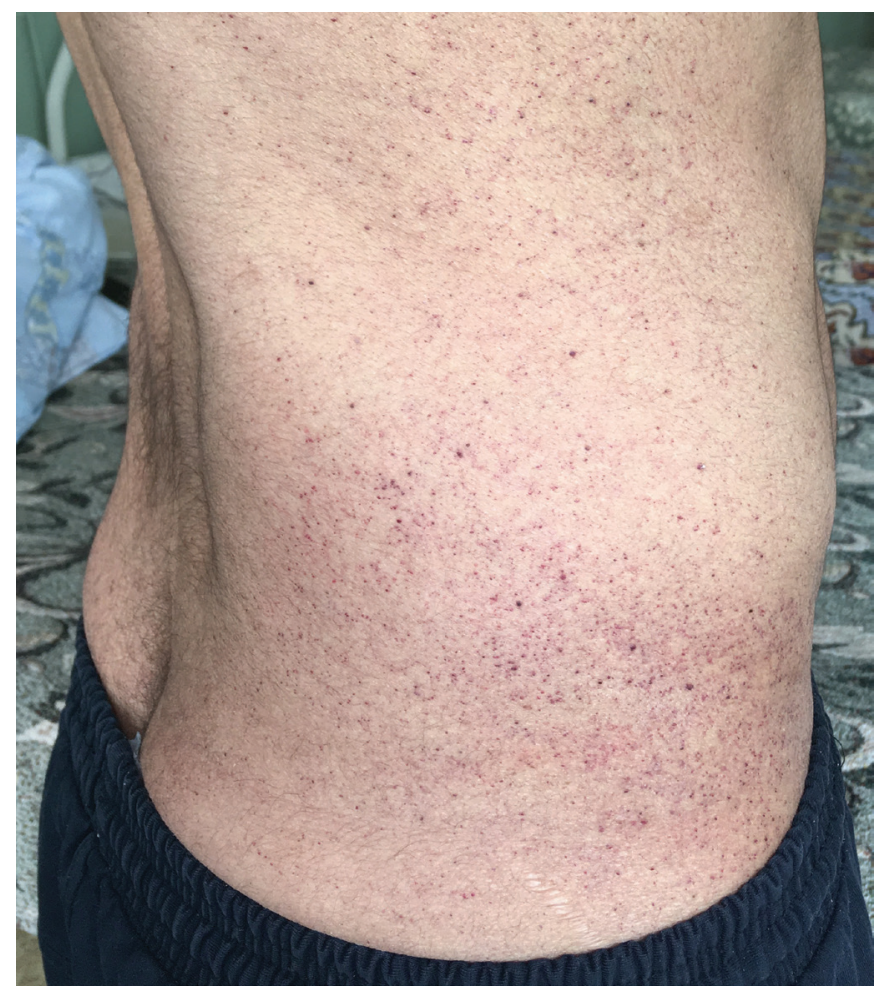

Figure 1 Multiple angiokeratomas in male patient with Fabry disease.

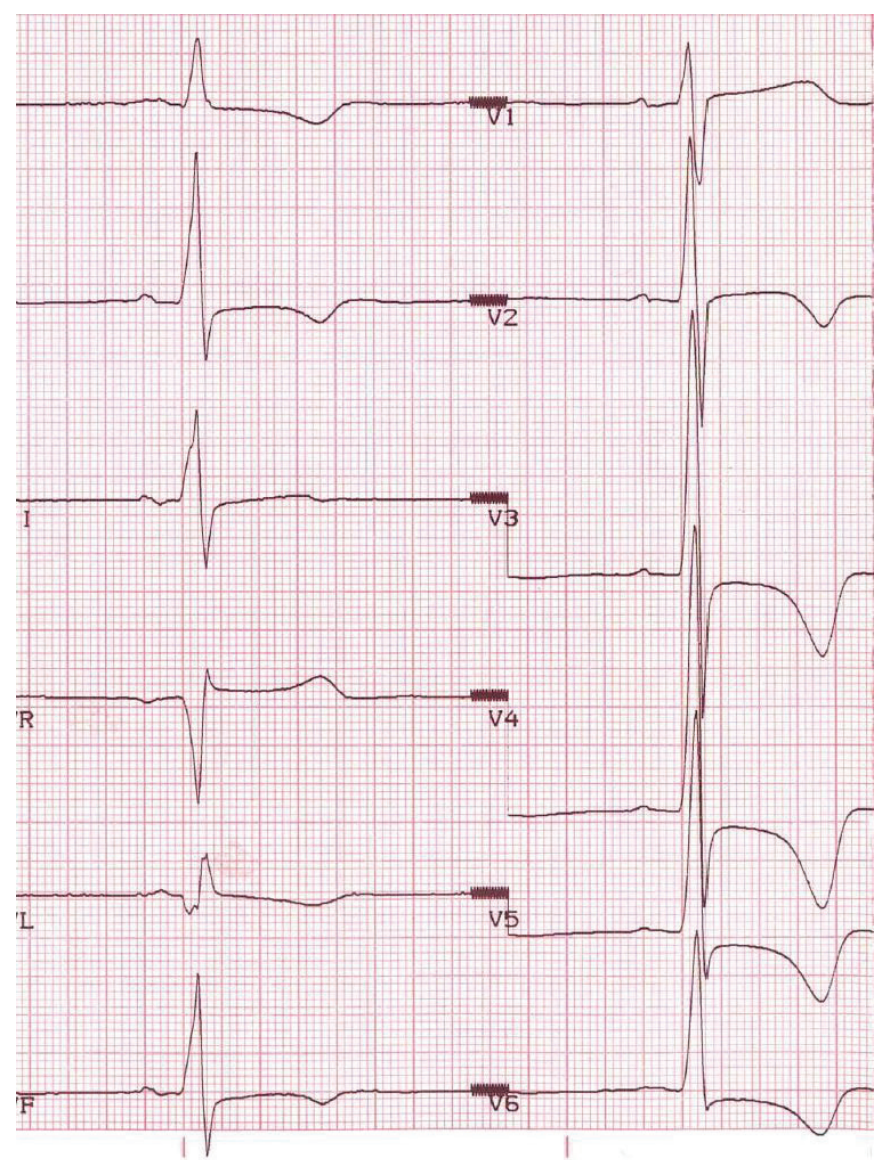

Figure 2 Significant left ventricular hypertrophy on ECG in the same patient.

We retrospectively studied the medical records of 107 adult patients with definite FD, including 70 males and 37 females aged 18 to 69 years (median 37 years). A total of 96 of 107 patients $(89.7 \%)$ had a history of classic phenotype of FD from childhood or adolescence. However, there was a significant delay to diagnosis of up to 51 years (median 18 years). Moreover, diagnosis was established by nationwide screening in dialysis units in $27.1 \%$ of patients or by family screening in $45.8 \%$ of patients. At the time of diagnosis, patients usually presented with a clinical picture of systemic disease with mild to moderate proteinuria with or without impairment of kidney function (86\%), left ventricular hypertrophy $(54.2 \%)$, white matter lesions on brain MRI (48.6\%) and/or a history of stroke (16.8\%).

A total of 28 of 107 patients (26.2\%) had previously at least one diagnosis of rheumatic disease (table 1). The common causes for referral to rheumatologist were skin rash and neuropathic pain. In 9 of 28 patients, the latter was initially misdiagnosed as joint pain. The possible causes of diagnostic errors included 'genuine' arthralgia $(\mathrm{n}=8)$, episodes of unexplained fever $(n=11)$, Raynaud phenomenon $(n=2)$ and the laboratory markers of inflammation $(n=12)$.

In summary, Vordenbäumen et al showed that in adult patients with joint pain and at least one swollen joint, FD is unlikely to be overlooked in the rheumatology practice. In contrast, our data suggest that every third patient with established FD receives at least one diagnosis of rheumatic disease, mostly in adolescence or at a younger age. In paediatric patients, the most common causes of incorrect diagnosis were angiokeratomas that can be confused with purpura and 


\begin{tabular}{ll}
\hline \multicolumn{2}{l}{ Table 1 'Rheumatic' diagnoses in patients with FD } \\
\hline Misdiagnoses & $\mathbf{n}(\%)$ \\
\hline Arthritis* $^{*}$ & $9(8.4)$ \\
Systemic vasculitis ${ }^{\prime}$ & $6(5.6)$ \\
Osler-Weber-Rendu disease & $5(4.7)$ \\
Systemic lupus erythematosus & $4(3.7)$ \\
Rheumatic fever & $4(3.7)$ \\
Familial Mediterranean fever & $1(0.9)$
\end{tabular}

*Arthritis included rheumatoid arthritis, juvenile rheumatoid arthritis and osteoarthritis.

tSystemic vasculitis included IgA vasculitis, Behcet disease, etc.

FD, Fabry disease.

pain in hands and feet, which was commonly interpreted as a manifestation of inflammatory arthritis. Moreover, approximately $10 \%$ of patients with FD presented with 'genuine' joint pain, episodes of unexplained fever and/or elevated markers of inflammation, that is, ESR and C reactive protein, which were usually regarded by physicians as indicators of rheumatic disease. Of note, hypohidrosis/anhidrosis is an important early warning symptom of $\mathrm{FD}$, which is frequently overlooked in clinical practice. Vortex keratopathy, or cornea verticillata, characterised by the whorl-like, linear opacities in the inferior part of the cornea is a relatively specific ocular sign of FD that can support diagnosis in more than half of the patients. In adult patients with a clinical picture of systemic disease involving heart, kidney, central and peripheral nervous system, and/or skin, FD can be misdiagnosed as systemic connective tissue disease or systemic vasculitis (particularly IgA vasculitis in the presence of skin rash or large-vessel vasculitis in patients with cerebrovascular events). In males, all manifestations of FD are more prominent and develop earlier than in females. A detailed family history is essential for the diagnosis of FD, while family screening in patients with established diagnosis usually identifies other affected family members who can benefit from earlier enzyme replacement therapy.

In primary care, 'don't expect to see a Zebra, when you hear hoof beats'. However, rheumatologists should be aware that it is not rare to have a rare disease and should recognise the zebras among the horses. ${ }^{4}$
Sergey Moiseev $\odot,{ }^{1}$ Ekaterina Karovaikina, ${ }^{1}$ Pavel I Novikov, ${ }^{1}$ Dilyara Ismailova, ${ }^{2}$ Alexey Moiseev, ${ }^{3}$ Nikolai Bulanov ${ }^{1}$

${ }^{1}$ Tareev Clinic of Internal Diseases, Sechenov First Moscow State Medical University, Moscow, Russian Federation

${ }^{2}$ Department of Orbital, Ophthalmic Plastic and Reconstructive Surgery, Scientific Research Institute of Eye Diseases, Moscow, Russian Federation

${ }^{3}$ Faculty of Medicine, Lomonosov Moscow State University, Moscow, Russian Federation

Correspondence to Professor Sergey Moiseev, Tareev Clinic of Internal Diseases, Sechenov First Moscow State Medical University, Moscow 119435, Russian Federation; clinpharm@mtu-net.ru

Contributors All authors participated in the preparation of the manuscript. Funding The study is funded by the Russian Academic Excellence Project 5-100.

Competing interests None declared.

Patient consent for publication Not required.

Provenance and peer review Not commissioned; internally peer reviewed.

(C) Author(s) (or their employer(s)) 2020. No commercial re-use. See rights and permissions. Published by BMJ.

\section{(A) Check for updates}

To cite Moiseev S, Karovaikina E, Novikov PI, et al. Ann Rheum Dis 2020;79:e71.

Received 2 April 2019

Accepted 7 April 2019

Published Online First 30 April 2019

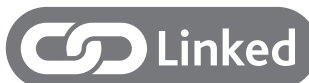

http://dx.doi.org/10.1136/annrheumdis-2019-215516

Ann Rheum Dis 2020;79:e71. doi:10.1136/annrheumdis-2019-215476

ORCID iD

Sergey Moiseev http://orcid.org/0000-0002-7232-4640

\section{REFERENCES}

1 Germain DP. Fabry disease. Orphanet J Rare Dis 2010;5.

2 Moiseev S, Fomin V, Savostyanov K, et al. The prevalence and clinical features of Fabry disease in hemodialysis patients: Russian nationwide Fabry dialysis screening program. Nephron 2019;141:249-55.

3 Vordenbäumen S, Brinks R, Richter JG, et al. Clinical characteristics of patients with alpha-galactosidase a gene variants in a German multicentre cohort of early undifferentiated arthritis. Ann Rheum Dis 2019;78:1286-7.

4 Evans WR, Rafi I. Rare diseases in general practice: recognising the zebras among the horses. Br J Gen Pract 2016;66:550-1. 